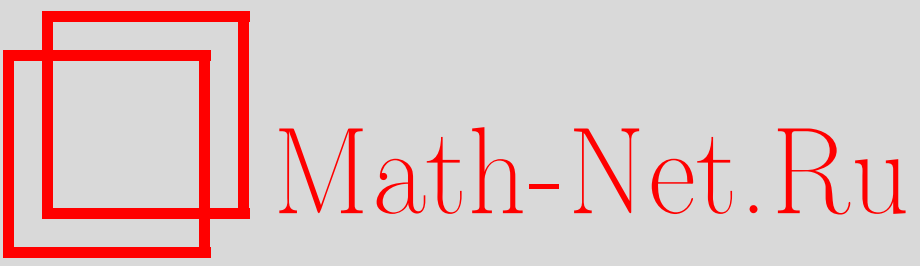

В. М. Максимов, Об одном обобщении кольца косых полиномов Оре, УМH, 2000, том 55, выпуск 4, 215-216

DOI: https://doi.org/10.4213/rm317

Использование Общероссийского математического портала Math-Net.Ru подразумевает, что вы прочитали и согласны с пользовательским соглашением

http://www.mathnet.ru/rus/agreement

Параметры загрузки:

IP: 3.85 .73 .92

26 апреля 2023 г., 09:01:46 


\title{
ОБ ОДНОМ ОБОБШЕНИИ КОЛЬЦА КОСЫХ ПОЛИНОМОВ ОРЕ
}

\author{
В. М. МАКСИмов
}

1. Напомним определение кольца косых полиномов (см. [1]-[4]).

Кольцо $A$ называется кольцом косых полиномов (левым) над кольцом $\mathscr{K}, \mathscr{K} \subset A$, если существует элемент $\tau, \tau \in A$, такой, что каждьй элемент $a, a \in A$, можно однозначно представить в виде $a=\alpha_{0}+\alpha_{1} \tau+\cdots+\alpha_{k} \tau^{k}$, где $\alpha_{i} \in \mathscr{K}, k=k(a)$.

Определение правого кольца косых полиномов аналогично. Ясно, что однозначно определяются выражения $\tau \alpha, \alpha \in \mathscr{K}$, называемые коммутациями, $\tau \alpha=\alpha_{0}^{(1)}+\alpha_{1}^{(1)} \tau+\cdots+\alpha_{m_{1}}^{(1)} \tau^{\left(m_{1}\right)}$, где $\alpha_{i}^{(1)} \in \mathscr{K}, m_{1}=m_{1}(\alpha)$. Коммутации определяют отображения $\psi_{j}^{(1)}: \psi_{j}^{(1)}(\alpha)=\alpha_{j}^{(1)}$; $j=0,1,2, \ldots$, которые в силу равенств $\tau(\alpha+\beta)=\tau \alpha+\tau \beta$ будут линейными отображениями кольца $\mathscr{K}$ в себя. Операторы $\left\{\psi_{j}^{(1)}\right\}$ обычно называются обобщенными диффференцированиями кольца $\mathscr{K}$. Между операторами $\left\{\psi_{j}^{(1)}\right\}$ существуют алгебраические выражения. Для их нахождения соотношением $\tau^{n} \alpha=\psi_{0}^{(n)}(\alpha)+\psi_{1}^{(n)}(\alpha) \tau+\cdots+\psi_{m_{n}}^{(n)}(\alpha) \tau^{m_{n}}$ определим операторы $\psi_{j}^{(n)}$, $n=1,2, \ldots$, на $\mathscr{K}$, где $\alpha \in \mathscr{K}, m_{n}=m_{n}(\alpha)$. Тогда операторы $\psi_{j}^{(n)}$ можно выразить через $\psi_{0}^{(1)}, \psi_{1}^{(1)}, \ldots$ (cм. [2], [5]).

Если $\psi(t)=\psi_{0}^{(1)}+\psi_{1}^{(1)} t+\psi_{2}^{(1)} t^{2}+\cdots$ есть производящий ряд для операторов $\psi_{m}^{(1)}$ с коммутирующей переменной $t$, то тогда легко получить, что

$$
\psi^{n}(t)=\psi_{0}^{(n)}+\psi_{1}^{(n)} t+\psi_{2}^{(n)} t^{2}+\cdots, \quad n=1,2, \ldots
$$

С другой стороны, из равенств $\tau^{m}(\alpha \beta)=\left(\tau^{m} \alpha\right) \beta ; \alpha, \beta \in \mathscr{K}, m=1,2, \ldots$, и единственности представлений $\tau^{n} \alpha$ имеем:

$$
\begin{array}{lll}
\psi_{0}^{(m)}(\alpha \beta)=\psi_{0}^{(m)}(\alpha) \beta+ & \psi_{1}^{(m)}(\alpha) \psi_{0}^{(1)}(\beta)+\psi_{2}^{(m)}(\alpha) \psi_{0}^{(2)}(\beta)+\cdots \\
\psi_{1}^{(m)}(\alpha \beta)= & \psi_{1}^{(m)}(\alpha) \psi_{1}^{(1)}(\beta)+\psi_{2}^{(m)}(\alpha) \psi_{1}^{(2)}(\beta)+\cdots \\
\psi_{2}^{(m)}(\alpha \beta)= & \psi_{1}^{(m)}(\alpha) \psi_{2}^{(1)}(\beta)+\psi_{2}^{(m)}(\alpha) \psi_{2}^{(2)}(\beta)+\cdots
\end{array}
$$

Операторы $\psi_{j}^{(m)}$ определяют матричное представление $\psi$ кольца $\mathscr{K}, \psi(\alpha)=\left\|\psi_{j}^{(m)}(\alpha)\right\|$, $\alpha \in \mathscr{K}$. Частный случай этого представления при $\psi_{0}^{(1)}=0$ был указан в [2].

2. Косые полиномы Оре [1] есть частный случай кольца косых полиномов с коммутацией

$$
\tau \alpha=\psi(\alpha)+\varphi(\alpha) \tau \quad \forall \alpha \in \mathscr{K} .
$$

В этом случае для операторов $\psi$ и $\varphi$ из (2) получим уравнения

$$
\text { 1) } \varphi(\alpha \beta)=\varphi(\alpha) \varphi(\beta), \quad \text { 2) } \psi(\alpha \beta)=\psi(\alpha) \beta+\varphi(\alpha) \psi(\beta) \text {, }
$$

которые называются условиями Оре для операторов $\psi$ и $\varphi$. Отображение $\psi$ есть $\varphi$-дифференцирование кольца $\mathscr{K}$. Условие (4) является необходимым и достаточным, чтобы существовало кольцо косых полиномов Оре с коммутацией (3). Доказательство можно найти в [3].

В случае произвольного кольца косых полиномов теорема существования также основана на выполнении равенств, вытекающих из условия ассоциативности.

ТЕОрема 1 (Условия существования). Пусть задано множество $\psi_{0}^{(m)}, \psi_{1}^{(m)}, \psi_{2}^{(m)}, \ldots$; $m=1,2, \ldots$, линейных отображений кольца $\mathscr{K}$ в себя. Тогда условия (1) и (2) являются необходимыми и достаточньми, чтобь существовало кольцо косых полиномов над ЖК с коммутацией

$$
\tau \alpha=\psi_{0}^{(1)}(\alpha)+\psi_{1}^{(1)}(\alpha) \tau+\cdots+\psi_{m_{1}}^{(1)}(\alpha) \tau^{m_{1}}, \quad m_{1}=m_{1}(\alpha) .
$$


3. Отличительное и важное свойство кольца косых полиномов Оре состоит в том, что, зная коммутации $\tau \alpha$ и $\tau \beta$, легко вычислить коммутацию $\tau(\alpha \beta)$. Мы рассмотрим более широкий случай кольца косых полиномов, в которых это свойство выполняется. А именно, рассмотрим кольцо косых полиномов, в котором

$$
\tau^{i} \alpha=\psi_{0}^{(i)}(\alpha)+\psi_{1}^{(i)}(\alpha) \tau+\cdots+\psi_{s}^{(i)}(\alpha) \tau^{s}, \quad i=1, \ldots, s .
$$

Мы будем называть коммутации вида (5) каноническими формами порядка $s$. Следовательно, в случае Оре имеется каноническая форма порядка 1. Колцо с каноническими коммутациями назовем каноническими.

Пусть $\mathscr{G}=\left\{g_{m}\right\}, m=1,2, \ldots$, есть некоторое множество образующих кольца $\mathscr{K}$. Тогда, полагая в (5) $\psi_{j}^{(i)}\left(g_{m}\right)=c_{m j}^{i} \in \mathscr{K}$, получим

$$
\tau^{i} g_{m}=c_{m 0}^{i}+c_{m 1}^{i} \tau+\cdots+c_{m s}^{i} \tau^{s}, \quad i=1, \ldots, s, \quad m=1,2, \ldots
$$

Очевидно, что по системе (6) однозначно восстанавливается система уравнений (5). С другой стороны, если элементы $\left\{c_{j k}^{i}\right\}$ задать произвольным образом, то кольцо, порождаемое соотношениями (6), может и не быть кольцом косых полиномов над $\mathscr{K}$. Поэтому естественно возникает вопрос: при каких соотношениях между элементами $\left\{c_{j k}^{i}\right\}$ для образуюших $\mathscr{G}$ соотношения (6) порождают какое-нибудь кольцо косых полиномов над $\mathscr{K} ?$

4. Определим на генераторах $\mathscr{G}$ отображение $\psi$ :

$$
\psi\left(g_{m}\right)=\left(\begin{array}{cccc}
g_{m} & 0 & \ldots & 0 \\
c_{m 0}^{1} & c_{m}^{1} & \ldots & c_{m s}^{1} \\
\ldots \ldots & \ldots \ldots \ldots \ldots \\
c_{m 0}^{s} & c_{m 1}^{s} & \ldots & c_{m s}^{s}
\end{array}\right)
$$

Если отображение (7) может быть продолжено до гомоморфизма кольца $\mathscr{K}$, то будем говорить, что для множества $\left\{c_{j k}^{i}\right\}$ и образующих $\mathscr{G}$ выполнено условие корректности. Далее, если при

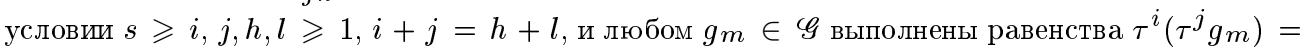
$\tau^{h}\left(\tau^{l} g_{m}\right)$, вычисленные из соотношений (6) при условии, что степени $\tau$ считаются линейно независимыми слева над $\mathscr{K}$, то мы будем говорить, что между множеством $\left\{c_{j k}^{i}\right\} \subset \mathscr{K}$ и множеством образующих $\mathscr{G}$ выполнено условие согласованности.

Теорема 2. Для того чтобы соотношения (6) порождали каноническое кольчо косых полиномов, необходимо и достаточно:

1. выполнения условия корректности для множеств $\left\{c_{j k}^{i}\right\}$ и $\mathscr{G}$;

2. выполнения условия согласованности между множествами $\left\{c_{j k}^{i}\right\}$ и $\mathscr{G}$;

3. для $i, j \geqslant 1, i+j \leqslant s$, и любого $g_{m} \in \mathscr{G}$ должснь иметь место равенства $\tau^{i}\left(\tau^{j} g_{m}\right)=\tau^{i+j} g_{m}$, где каждая коммутация вычисляется с помощью уравнениนั (6).

Наиболее эффективные следствия теоремы 2 получаются, когда кольцо $\mathscr{K}=\mathscr{F}[x]$ есть кольцо обычных полиномов над полем $\mathscr{F}$. В этом случае условия корректности выполняются автоматически.

\section{СПИСОК ЛИТЕРАТУРЫ}

[1] Ore O. // Ann. of Math. (2). 1933. V. 34. P. 480-508. [2] Smits T. H. M. // Indag. Math. 1968. V. 30. Р. 209-224. [3] Кон П. Свободные кольца и их связи. М.: Мир, 1975. [4] А ртамонов В. А. // УМН. 1998. Т. 53. № 4. С. 3-76. [5] Brungs H. H., Torner G. // J. Algebra. 1984. V. 87. P. $368-379$. 